
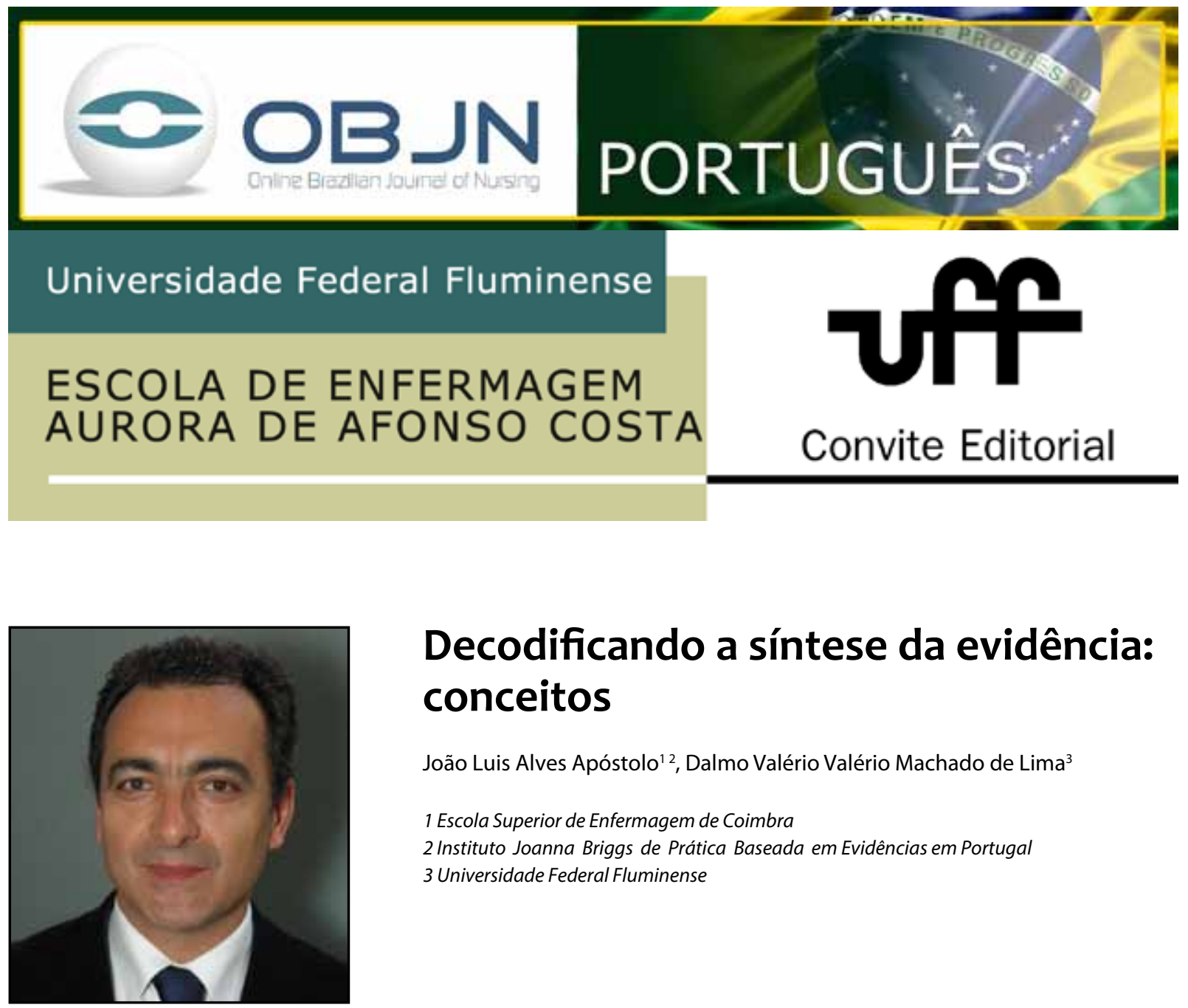

\title{
Decodificando a síntese da evidência: conceitos
}

\author{
João Luis Alves Apóstolo ${ }^{12}$, Dalmo Valério Valério Machado de Lima ${ }^{3}$ \\ 1 Escola Superior de Enfermagem de Coimbra \\ 2 Instituto Joanna Briggs de Prática Baseada em Evidências em Portugal \\ 3 Universidade Federal Fluminense
}

\section{RESUMO}

Etapa essencial à implementação da prática informada por evidências é a chamada síntese da evidência. Entretanto, sua concepção e aplicação é ainda distante dos pesquisadores, intituições de ensino e pesquisa, profissionais etc. Com o objetivo de facilitar a compreensão dos leitores acerca do tema, concatenando conceitos e prática, seguirão os editoriais de primavera e verão do OBJN. Uma das formas mais aceitas para desenvolver síntese da ciência é a revisão sistemática da literatura (RSL). Os métodos explícitos, sistemáticos e reprodutíveis utilizados na RSL têm por objetivo minimizar diferentes tipos de viés, incluindo a avaliação crítica da qualidade dos estudos que atendam aos critérios de inclusão da revisão em causa. Quando a evidência produzida gera recomendações para a prática ou política, a inclusão de evidências de baixa qualidade e com elevado risco de viés é problemática.

Descritores: Formatos de Publicação; Acesso à Informação; Enfermagem Baseada em Evidências. 
A prática informada por evidências (PIE) imprescinde de três elementos à sua real implementação ao longo do mundo, a saber: características pessoais, liderança e clima organizacional ${ }^{(1)}$.

Etapa essencial à aludida implementação da PIE é a chamada síntese da evidência. Entretanto, sua concepção e aplicação é ainda distante dos pesquisadores, intituições de ensino e pesquisa, profissionais etc. Assim, com o objetivo de facilitar a compreensão de seus leitores acerca do tema, concatenando conceitos e prática, seguirão os editoriais de primavera e verão do OBJN, respectivamente.

Dada a explosão do conhecimento, com cerca de dois milhões de novas entradas de publicações científicas em bases da literatura a cada ano, é difícil para os clínicos desenvolverem processos de síntese da ciência, pela perícia e recursos que um processo desta natureza exige. Por esta razão, devem existir equipes especializadas e treinadas para utilizar métodos rigorosos com vistas a reunir e avaliar todas as evidências disponíveis em determinados tópicos de interesse, aliviando os clínicos deste fardo(2).

Há vários fatores que podem motivar os autores a proceder à síntese de evidência: clarificação de evidências conflituantes, abordagem de questões cuja prática clínica é incerta, exploração de variações na prática, confirmação da adequação das práticas correntes, ou destaque de necessidade de investigações futuras ${ }^{(3)}$.

Uma das formas mais aceitas para desenvolver síntese da ciência é a revisão sistemática da literatura (RSL).. Há, assim, um número crescente de colaborações especializadas, institutos e centros com pessoal qualificado para treinar revisores, realizar revisões sistemáticas e facilitar a cooperação entre os colaboradores(2).

A RSL é uma investigação secundária complexa, detalhada e reprodutível que envolve um compromisso significativo de tempo e outros recursos. Nessa metodologia é utilizado um processo para localizar e sintetizar/agregar, a partir da literatura primária, todas as evidências existentes sobre um determinado tópico, e, para isso, o revisor usa uma fonte secundária de dados. A RSL é uma tentativa de integrar os dados empíricos, provenientes dos estudos primários, com a finalidade de descobrir a evidência internacional e produzir declarações que devem orientar a tomada de decisões clínicas. Requer, portanto, comunicação explícita e exaustiva dos métodos utilizados ${ }^{(2,3)}$.

Mesmo quando a evidência é limitada ou inexistente, as RSL resumem as melhores evidências disponíveis sobre um tema específico, fornecendo a melhor delas para subsidiar a tomada de decisões e para futuras necessidades de investigação clínica ${ }^{(4)}$.No caso de não existir informação primária sobre um determinado tópico, a opinião de peritos pode constituir a melhor evidência disponível ${ }^{(2)}$.

Os métodos explícitos, sistemáticos e reprodutíveis utilizados na RSL têm por objetivo minimizar diferentes tipos de viés, proporcionando, assim, resultados mais fiáveis que sustentem as conclusões e as decisões tomadas. A RSL é: uma metodologia reprodutível e explícita; uma procura sistemática que tenta identificar todos os estudos que satisfazem os critérios de elegibilidade; uma avaliação da validade das conclusões dos estudos incluídos, por exemplo, por meio da avaliação do risco de viés; uma apresentação sistemática e sintetizada das características e resultados dos estudos incluídos ${ }^{(2,4)}$.

Assim, para qualquer RSL deve desenvolver-se os seguintes passos: formular uma pergunta de revisão; definir critérios de inclusão e exclusão; localizar os estudos; selecionar os estudos; avaliar a qualidade metodológica dos estudos; extrair os dados; analisar/resumir e sintetizar os estudos relevantes; apresentar os resultados; interpretar os resultados; e determi- 
nar a aplicabilidade dos resultados ${ }^{(4)}$.

Para orientar uma revisão deverá ser previamente desenvolvido um protocolo orientadore ajustado à tipologia de revisão. Um protocolo de RSL deverá ser composto por diferentes seções, como o título, informações sobre a autoria, background, critérios de inclusão e exclusão para a seleção dos estudos, e por métodos de pesquisa para identificação de estudos, de avaliação crítica da sua qualidade, bem como de extração e síntese dos dados. No background devem ser apresentados os argumentos que sustentam a necessidade de desenvolver a revisão e definidos os conceitos em análise. Devem ainda ser colocados em contexto os diversos elementos da estratégia PICO ou da respetiva adaptação específica para cada revisão. A seção de métodos que especifica os critérios de inclusão e exclusão para a seleção dos estudos inclui o tipo de estudos, tipo de participantes, tipo de intervenções, tipo de medidas de resultado, sendo que os critérios a especificar podem variar dependendo do tipo de RSL planejada ${ }^{(4)}$.

Após realizar a RSL, os revisores apresentam um relatório cuja estrutura deverá ser inerente ao desenvolvimento das seções do protocolo. Ele deverá conter, genericamente: (i) dados respeitantes ao risco de viés dos estudos incluídose decorrentes do processo da análise da qualidade metodológica; (ii) descrição de estudos incluídos na revisão; (iii) resultados que dão resposta à questão de revisão; (iv) discussão dos resultados, indicação das fragilidades da revisão e dos estudos incluídos, bem como as implicações para a prática e para a investigação; e, finalmente, (v) uma conclusão relativa à questão de revisão(4).

Um passo crítico em qualquer RSL é a avaliação crítica da qualidade dos estudos que atendam aos critérios de inclusão da revisão em causa. A noção de métodos para minimizar o viés e estabelecer a credibilidade nas revisões sistemáticas tem sido amplamente desenvolvida e debatida em termos de evidência quantitativa e qualitativa.

O objetivo da avaliação crítica é estimar até que ponto os riscos potenciais de viés e de consistência da qualidade foram minimizados durante a conceptualização e a realização dos estudos primários individuais, e se há utilização adequada do método e da metodologia. Cada estudo elegível deve ser avaliado com base num conjunto de critérios para estabelecer a validade e a confiabilidade do processo e dos resultados. Esta avaliação crítica deve ser apresentada no relatório, na seção de resultados da revisão, sendo acompanhada de uma discussão relativa à qualidade metodológica e ao potencial risco de viés de todos os estudos incluídos e excluídos com base neste juízo crítico.

Quando a evidência produzida gera recomendações para a prática ou política, a inclusão de evidências de baixa qualidade e com elevado risco de viés é problemática. Nas revisões preconizadas pelo Joanna Briggs Institute (JBI) a equipe de revisores pode definir um ponto de corte que oriente a decisão de excluir estudos considerados de baixa qualidade ou com alto risco de viés. Pode considerar, contudo, abordagens alternativas, tais como meta-regressão ou análise de sensibilidade, no caso de haver lugar para a meta-análise ${ }^{(4)}$.

As evidências sobre os efeitos das intervenções podem ser constituídas a partir de estudos com risco diversificado de viés. Há duas abordagens que são igualmente razoáveis na condução de uma revisão sistemática de eficácia. A de incluir somente estudos com baixo ou moderado risco de viés, justificando como este risco é determinado e o que se considera como o grau de risco de viés e excluindo todos os estudos considerados de alto risco. $\mathrm{E}$ a de incluir todos os estudos, independentemente do seu risco de viés, e explicitamente considerar o risco dos diferentes estudos durante a síntese de dados ${ }^{(5)}$. 
A avaliação da qualidade dos estudos quantitativos de eficácia incluídos numa revisão deve enfatizar o risco de viés dos seus resultados, ou seja, o risco de superestimar ou subestimar o efeito da intervenção ${ }^{(3)}$. O viés não deve ser confundido com imprecisão. O primeiro refere-se ao erro sistemático, enquanto a precisão refere-se ao erro aleatório e reflete-se no intervalo de confiança em torno da estimativa de efeito da intervenção de cada estudo e no peso dado aos resultados de cada estudo numa meta-análise. Os resultados mais precisos têm mais peso na meta-análise ${ }^{(3,4)}$.

A colaboração Cochrane estabelece a diferença entre risco de viés e qualidade metodológica. O termo avaliação da qualidade metodológica tem sido utilizado para fazer referência à avaliação crítica dos estudos incluídos e sugere que o estudo foi desenvolvido sob os mais elevados padrões metodológicos ${ }^{(3)}$.

Um estudo pode ser realizado com os mais altos padrões possíveis de qualidade, mas ter um elevado risco de viés relacionado, por exemplo, com a impraticabilidade para ocultar aos participantes a intervenção a que estão a ser sujeitos. Esta é, de fato, uma condicionante das intervenções não farmacológicas comparadas com os cuidados usuais (exemplo da estimulação cognitiva), contrariamente a intervenções farmacológicas sobre as quais é possível fazer estudos cegos com um fármaco e um placebo. Outro exemplo é o de uma intervenção cirúrgica em que é impossível ocultar essa intervenção. Assim, é inadequado julgar estes estudos como tendo baixa qualidade, não significando, contudo, que estão livres de viés resultante do conhecimento pelos participantes da intervenção a que estão sujeitos ${ }^{(3)}$.

O risco de viés nos resultados de cada estudo, que contribui para uma estimativa do efeito, é um dos vários fatores que devem ser considerados ao avaliar a qualidade de um corpo de evidência. Nos estudos de eficácia, o controle do risco de viés orienta-se para as características dos estudos em relação à seleção, intervenção, detecção, atrito, e publicação, entre outros.

Também nas RSL de estudos qualitativos se justifica a síntese da evidência porque os resultados a partir de um único estudo qualitativo não devem ser considerados para produzir recomendações para a prática ${ }^{(4)}$. Também, tal como na investigação quantitativa, a avaliação crítica dos estudos primários qualitativos é fundamental para estabelecer a sua qualidade(4,6).

Tradicionalmente, os termos usados para avaliar criticamente o rigor da investigação são a confiabilidade (reliability) e a validade. Geralmente, a confiabilidade é a medida em que os resultados de um estudo relativos a uma medição são reprodutíveis em diferentes circunstâncias. Por sua vez, a validade refere-se ao grau em que um estudo reflete ou avalia o conceito específico que o investigador está a tentar medir com precisão. Mas a avaliação crítica de estudos qualitativos apresenta contornos diferentes. Questões de natureza ontológica, epistemológica, metodológica, ética, afiliação, background, experiência e contexto do investigador são fundamentais para aferir o valor da investigação qualitativa primária e podem fazer da síntese dos resultados qualitativos uma tarefa complexa e assustadora ${ }^{(4,6,7)}$.

A confiabilidade/reprodutividade das medidas, a validade interna e externa e a objetividade são critérios considerados essenciais para a qualidade de estudos quantitativos. Nos estudos qualitativos devemos considerar critérios correspondentes. Neste sentido, os conceitos de dependability, credibility, transferability e confirmability, apontados por Lincoln e Guba(8) deverão ser considerados para estabelecer o valor de um estudo qualitativo.

\section{REFERÊNCIAS}


1. Apóstolo J, Cardoso D, Rodrigues MA. It takes three to tango: embracing EBP. JBI Database System Rev Implement Rep [internet]. 2016 Apr [cited xx xx xx]; 14 (4): 1-2. Available from: http:// journals.lww.com/jbisrir/Fulltext/2016/04000/lt_ takes_three_to_tango__embracing_EBP.1.aspx

2. Pearson $A$, White $H$, Bath-Hextall $F$, Salmond $\mathrm{S}$, Apóstolo J, Kirkpatrick P. A mixed-methods approach to systematic reviews. International Int J Evid Based Healthc [internet]. 2015 [cited Xx xx xx]; 13(3: 121-131. doi: 10.1097/ XEB.0000000000000052.

3. Higgins JP, Green S. Cochrane handbook for systematic reviews of interventions, version 5.1.0. Londres: The Cochrane Collaboration; 2011. [cited xx xx xx]. Available from: http://handbook. cochrane.org/

4. Joanna Briggs Institute. Joanna Briggs Institute reviewers' manual: 2014 edition. Adelaide: The Joanna Briggs Institute; 2014. [cited xx xx xx]. Available from: http://joannabriggs.org/assets/ docs/sumari/reviewersmanual-2014.pdf

5. Tufanaru C, Munn Z, Stephenson M, Aromataris $E$. Fixed or random effects meta-analysis? Common methodological issues in systematic reviews of effectiveness. Int J Evid Based Healthc. 2015; 13(3): 196-207.

6. Sandelowski $\mathrm{M}$, Voils $\mathrm{Cl}$, Barroso J. Defining and designing mixed research synthesis studies. Res Sch. 2006; 13(1), 29-40.

7. Apostolo J, Gameiro M. Referências onto-epistemológicas e metodológicas da investigação em enfermagem: uma análise crítica. Revista Referência. 2005; 2(1), 29-38.

8. Lincoln YS, Guba EG. Naturalistic Inquiry. Newbury Park, CA: Sage Publications; 1985.

Recebido: 09/08/2016

Aprovado: 09/08/2016 